\title{
Carbon Quantum Dots: A Safe Tool to Learn about Quantum Phenomenon in Nanomaterials
}

\author{
A. A. C. Cruz ${ }^{1}$, R. M. Freire ${ }^{1, *}$, S. V. Carneiro ${ }^{1}$, O. P. Ferreira ${ }^{2}$, P. B. A. Fechine ${ }^{1}$ \\ ${ }^{1}$ Grupo de Química de Materiais Avançados (GQMAT), Departamento de Química Analítica e Físico-Química, Universidade Federal do \\ Ceará - UFC, Campus do Pici, Fortaleza, CE, Brazil \\ ${ }^{2}$ Laboratório de Materiais Funcionais Avançados (LaMFA), Departamento de Física, Universidade Federal do Ceará - UFC, Campus do \\ Pici, Fortaleza - CE, Brazil
}

\begin{abstract}
In this paper, a lab experiment to demonstrate quantum phenomena was developed using carbon quantum dots (CQDs), a new carbon-based fluorescent nanomaterial, discovered in 2004. Therefore, a practical class was developed to demonstrate quantum phenomena using cheap and safe chemical products. Over 140 students from biotechnology, pharmacy, engineers and geology courses have successfully performed the proposed lab experiment. By following a short, easy and totally safe procedure, the students were able to synthesize CQDs, as well as to visualize the quantum phenomena related to the light scattering, absorption and emission. It was also possible to obtain the absorption spectrum of the CQD sample, with an absorption band at $325 \mathrm{~nm}$. Additionally, a wavelength-dependent behavior was observed for the synthesized CQDs. This may be used to deeply study quantum confinement effects. Therefore, CQDs can be a powerful and versatile tool to learn about quantum phenomena in nanomaterials.
\end{abstract}

Keywords Inorganic Chemistry, Laboratory Instruction, Synthesis, Quantum Chemistry

\section{Introduction}

About a decade ago, $\mathrm{Xu}$ et al [1] accidentally discovered a new luminescent nanoparticles (NPs): the carbon quantum dots (CQDs). Since them, the growing interest in the use of this nanomaterial has been emerging. In the last years, CQDs have attracted much attention because their fluorescent and biological properties. For instance, CQDs show biocompatibility, a required characteristic for application in nanomedicine [2]. The fluorescence intensity aligned to the nanoscale size makes CQDs useful for imaging [3]. As a consequence of these characteristics, a huge amount of publications exploring CQDs in different fields can be found in the literature [4-6]. However, the potential of CQDs in the educational field is still unknown given the low number of investigations [7]. In this sense, it is important to highlight the optical properties (absorption/emission) of CQDs. Many papers report that the emission can be excitation-dependent [8-11] or independent [12-14]. As a mechanism of the luminescence of CQDs may involves surface traps and quantum effects [15-17], CQDs may serve as a promising candidate to demonstrate quantum-related phenomenon for students. This places these new NPs as a valuable learning tool.

* Corresponding author:

rafael.m.freire@gmail.com (R. M. Freire)

Published online at http://journal.sapub.org/Jlce

Copyright (@) 2017 Scientific \& Academic Publishing. All Rights Reserved
Beyond CQDs, semiconductor nanocrystal quantum dots (QDs) such as CdSe can also be considered a powerful education tool [18]. Their size-dependent optical properties are perfect to allow students to visualize applications of quantum chemistry. However, to study quantum phenomenon through of QD becomes a problem, since it involves the use of heavy metals and toxics. This poses a danger for students. In addition, these types of metals are a serious source of pollution in the biosphere, which significantly affects the ecological quality of the environmental $[19,20]$. The effects of heavy metals on human health have been extensively investigated. It is well-known an exposure to these metals can contribute for a broad spectrum of carcinogenic, mutagenic and/or toxicological effects [21-23]. In this regard, cadmium (Cd) has been classified as a potential human carcinogen metal by U.S. Environmental Protection Agency. Moreover, it can cause kidney dysfunction or even death when at high levels concentration in the human body [24, 25]. Another negative factor is the methodology employed to synthesize the QDs. The students have to deal with dangerous chemicals, including sodium borohydride $\left(\mathrm{NaBH}_{4}\right)$, for example [26]. Taken together, the use of QDs in a lab experiment to show students quantum phenomena is not totally safe.

Given the exposed, imagine a lab experiment developed to demonstrate quantum phenomena using inexpensive and safe chemicals. This may be possible with CQDs. Besides new, the literature already reported many methods to synthesize these luminescent NPs. It can be cited 
electrochemical [27], combustion [28], hydrothermal [29], microwave [30], ultrasound [31], among others. Although, the synthesis of CQDs through combustion is simple, safe and cheap chemicals are used. For instance, Dong et al. [32] have easily synthesized CQDs via pyrolysis of citric acid at $180^{\circ} \mathrm{C}$. Recently, the synthesis of CQD has also been reported in the literature using citric acid as precursor [33]. Commercial products like milk [34], gelatin [35] and Nescafe $^{\circledR}$ [36] can play as a carbon source to obtain CQDs. Another sources such as hair fiber [37], orange [10] or banana [38] juice or even sugars [39, 40] are also reported in the literature. As it can be seen, all "chemicals" cited as a source of carbon are cheap and easy to find, which further places the CQDs as a safe and potential nanomaterial to compound a lab experiment for students. In this paper, we have developed a simple lab experiment to demonstrate quantum phenomena using CQDs. The preparation method of this nanomaterial is totally safe for students. No dangerous chemicals are used. Basically, the students performed the partial decomposition of the citric acid to obtain the CQDs. The experiment took no longer than $90 \mathrm{~min}$ and the students were allowed to observe absorbance/emission phenomena, as well as the light scattering.

\section{Design}

\subsection{Chemicals and Materials Required}

The chemical utilized in this study was only citric acid anhydrous $\left(\mathrm{C}_{6} \mathrm{H}_{8} \mathrm{O}_{7}, 99.5 \%\right.$, Vetec $)$. This chemical was used as received. In order to perform the procedure, the following materials were required:
a. $50 \mathrm{~mL}$ beaker
b. muffle furnace
c. 3 in 1 Laser Light Pointer (laser diode, wavelength 630-650 nm; output < 5mW);
d. cuvette
e. UV-Vis Spectrophometer

\subsection{Procedure}

Initially, $500 \mathrm{mg}$ of citric acid anhydrous was weighted in a $50 \mathrm{~mL}$ beaker. Subsequently, the beaker containing the citric acid was leaded for a muffle furnace Linn Elektro Therm, which it was previously heated at $180^{\circ} \mathrm{C}$. Right after put the beaker, the students set up a timer for $30 \mathrm{~min}$. During this time, the instructor briefly explained some quantum phenomenon-related points (absorption, emission, fluorescence and phosphorescence), as well as the Tyndall effect. After this time, the beaker was carefully removed and allowed to reach the room temperature naturally. Then, 40 $\mathrm{mL}$ of distillated water was added to disperse the CQDs. Next, a red Laser $(630-650 \mathrm{~nm})$ was positioned to goes through the solution to check the presence of the CQDs using the Tyndall effect. Comparatively, the same procedure was also evaluated using only the solvent (distillated water). Afterwards, the solution was exposed to the short-wavelength ultraviolet lamp (365 nm) to observe the emission coming from the synthesized nanomaterials. Then, an aliquot of $1 \mathrm{~mL}$ of CQDs dispersion was first diluted (1:50) and $2 \mathrm{~mL}$ of this dispersion was transferred to a glass cuvette $(1 \mathrm{~cm})$ to acquire the absorption spectrum $(300-600 \mathrm{~nm})$ at room temperature using a Spectrophometer model UV-1800 from Shimadzu equipped with deuterium and tungsten lamp. A schematic picture illustrating the timeline of the lab experiment proposed is displayed in the Fig. 1.

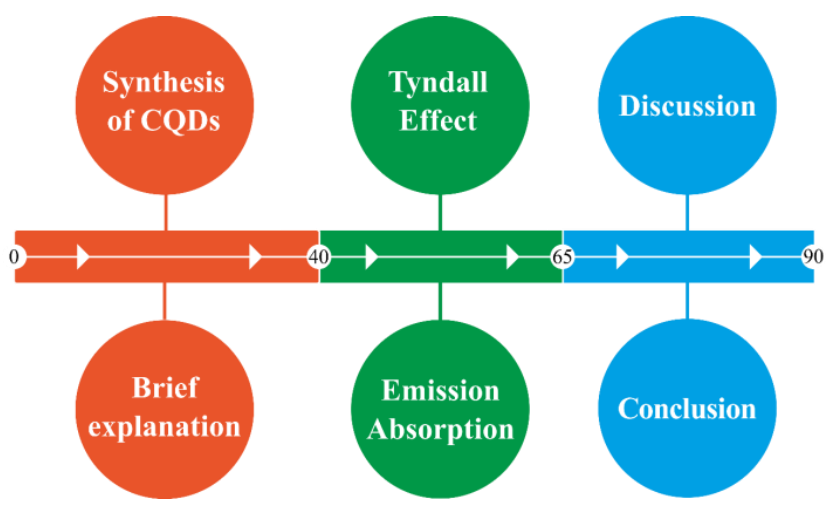

Figure 1. Course of time for the lab experiment proposed in this work. The experiment was divided into three blocks. The first block takes $40 \mathrm{~min}$, while the second and third $25 \mathrm{~min}$ each. This is also represented by orange, green and blue colors, respectively. The words inside the circles are the subject what was explained in each block

Since it was not used dangerous chemicals, only standard laboratory safety precautions must be followed. Therefore, protective clothing, appropriates gloves and goggles were worn to carried out any part of the procedure.

\section{Results}

\subsection{Synthesis of CQDs}

145 undergraduate students from geology, biotechnology, engineers and pharmacy have successfully obtained CQDs (Fig. 2) in the lab class. Fig. 2(a) shows the starting material (citric acid) inside a $10 \mathrm{~mL}$ glass vial under white light. Once this material has a crystalline structure, following the pathway to synthesize CQDs, the initial circle (first white square) presents a part of the citric acid unit cell built using crystal structure data from the Cambridge Structural Database (CSD - number 635772). The software utilized to visualize the cell unit was the CrystalMaker ${ }^{\circledR}$, version 2.5.1. Following the white arrows, the temperature is increased and it is expected that citric acid to melt at $153^{\circ} \mathrm{C}$. Thus, the circle related to the second white square denotes citric acid molecules in a liquid state. Subsequently, the temperature is raised up to $180^{\circ} \mathrm{C}$ an incomplete thermal decomposition proceeds. In this chemical reaction, the mechanism of the formation of the CQDs involves the condensation of citric acid molecules into $\mathrm{sp}^{2}$ domains. This is illustrated at circle linked to the third white square. Beyond, water molecules from condensation reactions and citric acid molecules are still present. The software ChemSketch, version for students, 
was used to build the water molecules. Afterwards, it is expected these $\mathrm{sp}^{2}$ nucleus formed to perform crosslinking and stacking as the reaction proceeds [41]. Therefore, the circle linked to the fourth white square shows large $\mathrm{sp}^{2}$ domains, as well as water and citric acid molecules. Both entities presented at the circles related to the third and fourth white square are expected to have $-\mathrm{OH}$ and $-\mathrm{COOH}$ groups on the surface. Since the carbon source used to synthesize the CQDs was the citric acid. These chemical functions were hidden in the illustration. Finally, Fig. 2(b) shows the as-synthesized CQDs under white light. Clearly, an orange compound can be seen.

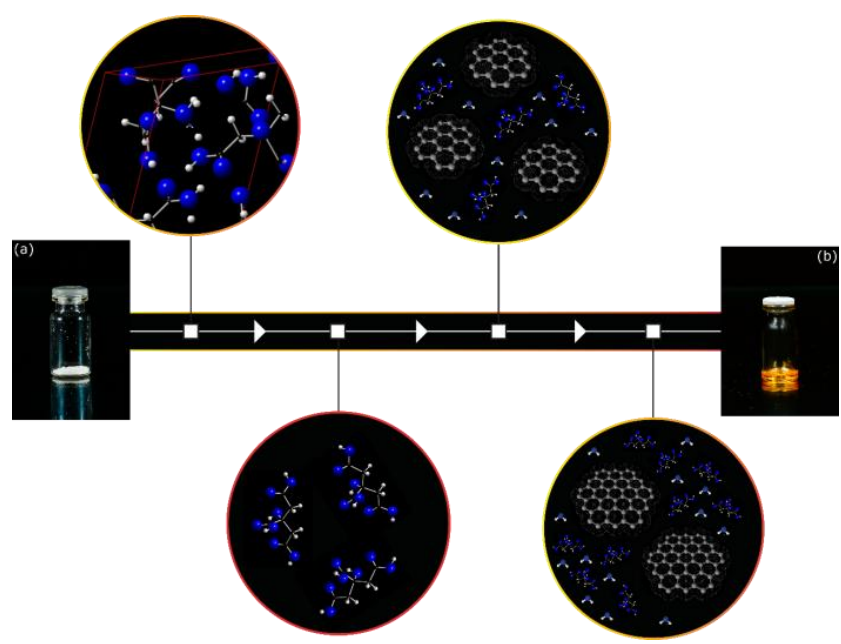

Figure 2. Schematic illustration of the thermal decomposition process to obtain CQDs. The pictures (a) and (b) displays citric acid inside $10 \mathrm{~mL}$ glass vial before and after the thermal process, respectively. The gray, blue and white balls denote the elements carbon, oxygen and hydrogen, respectively

\subsection{Tyndall Effect and Emission}

Once the CQDs were synthesized and cooled down naturally, the next step of the lab experiment proposed in this work was to check the Tyndall effect and emission. For this purpose, the whole amount of the CQDs was first dispersed in $40 \mathrm{~mL}$ of distilled water. Then, in order to check the presence of nanomaterials, the red Laser $(630-650 \mathrm{~nm})$ was pointed to the colloidal dispersion and a Laser going through the liquid (Fig. 3(a)) was observed by the students. By definition, the Tyndall effect is a phenomenon that occurs due to the interaction of light and particles that constitute colloidal systems. In this effect, the electromagnetic wave is spread causing a change in the direction of light. Taking this into account, the presence of the CQDs in the liquid was easily evidenced. Since the studied system was able to scatter the electromagnetic wave in all directions. For comparison, a beaker containing only distillated water was also unilaterally illuminated using the red Laser. In this experiment, absence of red Laser was observed in the liquid, which confirms the absence of the nanomaterials. Furthermore, Fig. 3(a) also displays a colloidal dispersion containing the CQDs under white light. Except by Tyndall effect, no additional quantum phenomenon can be observed in this condition.
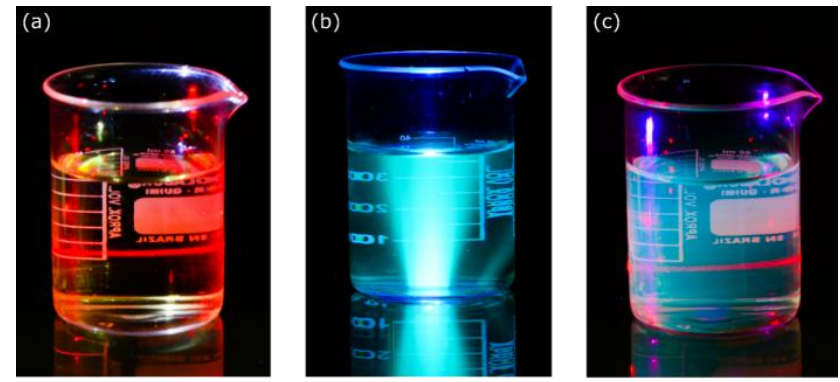

Figure 3. Demonstration of quantum phenomenon related to the emission and scattering of the light. (a) Front side view of the light scattering result of the red Laser going through the dispersion colloidal containing the CQDs synthesized. (b) Spontaneous blue emission of the dispersion colloidal while is illuminated from down side using black lamp. (c) Both spontaneous emission and light scattering of the red Laser. In this case, the 3 in 1 pointer was positioned at the left side of the recipient

Upon demonstration of the light scattering, the ultraviolet lamp $(365 \mathrm{~nm})$ of the 3 in 1 Laser light pointer was positioned to goes through the colloidal dispersion and a blue light was seen by students. Spontaneous emission of radiation from a photoexcited species can be specifically considered photoluminescence $[42,43]$. Therefore, the blue light coming out from the colloidal dispersion containing CQDs (Fig. 3(b)) may lead the students to considerate this quantum phenomenon. However, it can be further classified as fluorescence or phosphorescence. Traditionally, lifetime is used as a classification parameter to identify the types of photoluminescence from a phenomenological point of view. In this regard, a short-lived luminescence is considered fluorescence, while a long-lived is phosphorescence. Once no emission was observed upon removal of the UV lamp, the phenomenon was further classified as fluorescence. In order to further demonstrate of the quantum phenomena, Fig. 3(c) shows both fluorescence and Tyndall effect.

\subsection{Absorption Spectra}

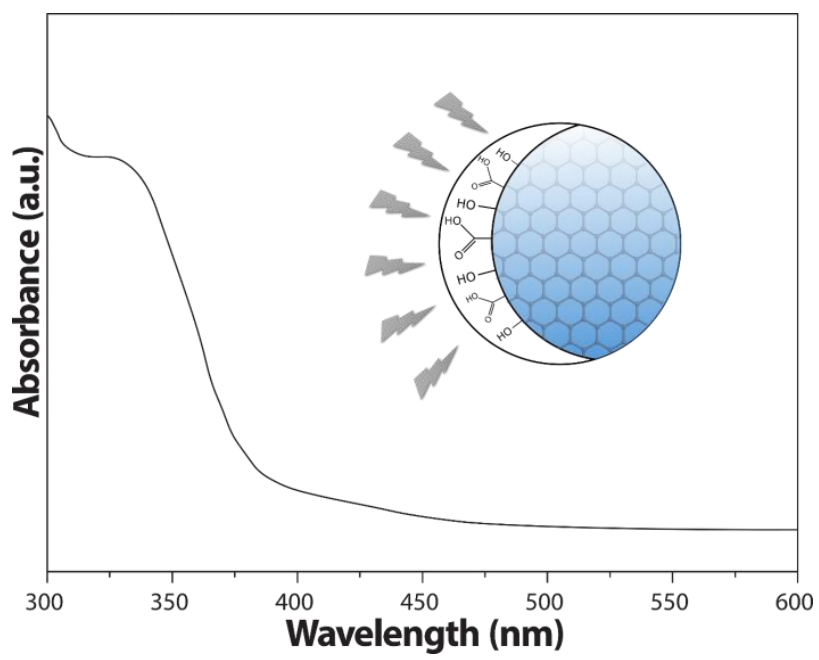

Figure 4. UV-Vis absorption spectrum recorded at room temperature. The illustration inside the graph denotes the irradiation of CQDs presenting $-\mathrm{OH}$ and $-\mathrm{COOH}$ groups on the surface, as well as the $\mathrm{sp} 2$ domains in the core 
Once emission and scatter of the light was demonstrated, the next quantum phenomenon to be studied was absorption. In this regard, an aliquot of $1 \mathrm{~mL}$ of the diluted colloidal dispersion containing the CQDs was placed in a glass cuvette and an absorption spectrum was recorded. Fig. 4 displays the spectrum of the CQDs in the range of $300-600 \mathrm{~nm}$. Clearly, there is a very broad absorption band centered at $325 \mathrm{~nm}$. Given the results, the quantum phenomenon of light absorption was easily demonstrated for the students using the proposed nanomaterials.

\section{Discussion}

A simple lab experiment involving absorption, emission and scattering of the light was developed and applied for 145 undergraduate students. The lab experiment in this work begins with the students performing an easy procedure to synthesize the CQDs. Thus, the product after the thermolysis (see Fig. 2(b)) was suspended in $40 \mathrm{~mL}$ distillated water. As a consequence, a pale yellow liquid system was obtained. The light scattering through Tyndall effect was first demonstrated. In this sense, the red Laser $(630-650 \mathrm{~nm})$ of the 3 in 1 pointer was positioned to pass through the liquid to check the presence of the nanomaterials. The Laser was observed in the liquid system, which confirms the presence of the CQDs. In addition, the experiment can be helpful to classify this liquid system as a colloidal dispersion where the NPs size should be in the range $1-1000 \mathrm{~nm}$ [44]. To further support our consideration, it was also performed TEM for the synthesized CQDs. The micrograph can be seen in the Fig. 5(a). Clearly, the CQDs formed agglomerates. This may contribute to the light scattering. However, the Fig.5(b) reveals particles with average diameter of $4.1 \pm 1.1$. nm, which confirms the existence of a colloidal suspension.
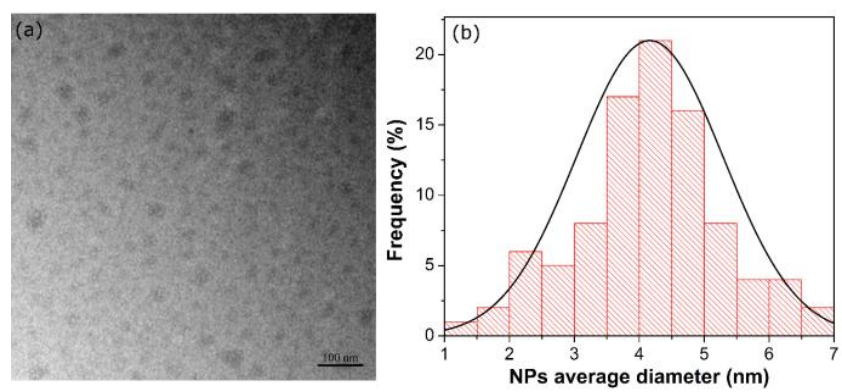

Figure 5. (a) TEM micrograph of the synthesized CQDs. (b) Distribution size graph built using over 90 NPs randomly selected

Afterwards, the UV lamp ( $365 \mathrm{~nm}$ ) of the 3 in 1 red Laser pointer was positioned to goes through the liquid. A blue light coming out from the dispersion could be observed by the students, which demonstrated the emission of the light from the nanomaterials (Fig. 3(b)). As luminescent carbon-based NPs, CQDs are able to emit radiation during exposure to the short-wavelength UV lamp. Therefore, considering a basic mechanism (Fig. 6), it can be mentioned the electrons in CQDs were first excited from an orbital in the ground state (HOMO) to a high energy orbital (LUMO) through the absorption of the radiation. Next, the de-excitation proceeds and the electrons should return to the ground state. During this process, spontaneous emission of radiation can be observed as a way to discard the excess of the energy to stabilize the quantum system. Besides this basic chemistry, the instructor may briefly cite other photoluminescence mechanisms related to the band gap transitions between $\pi$-domains or defects on the surface of CQDs [45-47]. The fact of the photoluminescence mechanism remains unclear can further lead the students to understand chemistry as a building science.
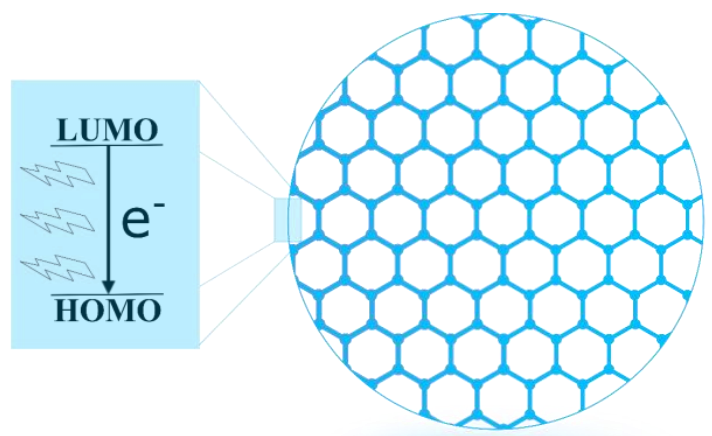

(b)

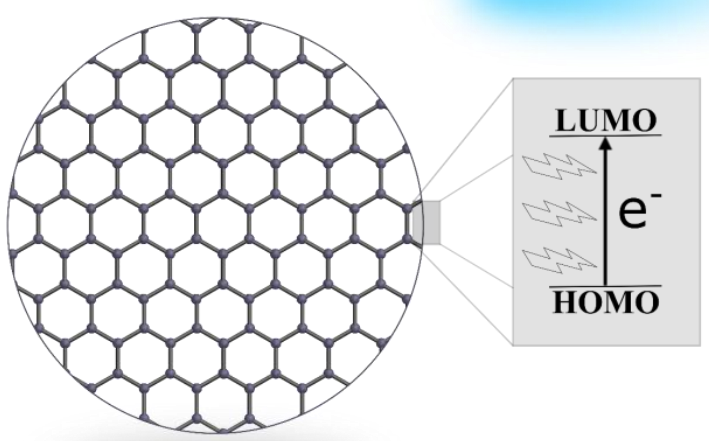

(a)

Figure 6. Schematic illustration representing the basic mechanism to describe photoluminescence. Once the absorption process (a) does not present spontaneous emission, the excitation of the electron ( $\mathrm{e}^{-}$) is colorless. By contrast, blue light can be seen as a result of the de-excitation process, i.e. emission process (b)

The absorption of the light was demonstrated using a UV-Vis spectrophotometer. A broad absorption band at 325 $\mathrm{nm}$ According to the preview studies $[48,49]$ this is a typical profile for CQDs where the absorption band observed can be attributed to the $\mathrm{n}-\pi^{*}$ transition coming from $\mathrm{C}-\mathrm{O}$ bonds. Usually, bands below $300 \mathrm{~nm}$ can be related to the $\pi-\pi^{*}$ transition of aromatic domains [29, 50]. However, the instructor may also lead the attention of the students for the high absorbance below $325 \mathrm{~nm}$. It seems to have other groups in the CQDs also capable to absorb the light. Therefore, the students can realize how different groups interact to the light, even observing at one single nanoparticle. 
The lab experiment performed in this work was built based on undergraduate lab without equipment to check the fluorescence intensity. Obviously, the presence of fluorimeter in the undergraduate chemistry laboratory allows the students to visualize the emission under different excitation wavelength, for example. Fig. 7 shows the as-synthesized CQDs fluorescence intensity recorded using excitation wavelengths in the range of $325-405 \mathrm{~nm}$. As it can be seen, a wavelength-dependent behavior was found. According to the literature, this can be related to the NPs size [51] and/or energy traps created by surface groups [52].

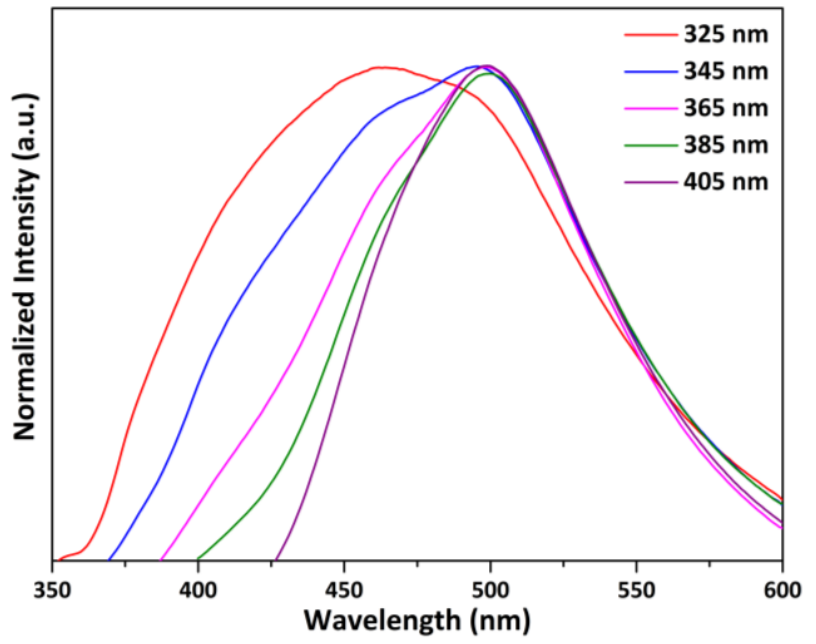

Figure 7. Normalized fluorescence intensity of the as-synthesized CQDs recorded at room temperature from 350 to $600 \mathrm{~nm}$ using different excitations wavelength $(325-405 \mathrm{~nm})$. The normalization was performed dividing each $\mathrm{Y}$-axis value by the maximum $\mathrm{Y}$-axis value observed

Thus, the demonstration of quantum phenomenon is not an easy task to be performed in a simple lab experiment. For instance, QDs seems to be a perfect material to allow students visualize absorption/emission of the light, as demonstrated by Landry (2014) [18]. However, it is still danger for students to deal with heavy metals like Cd. Only citric acid was used as a carbon source in the lab experiment developed in this work. Therefore, by following standard precautions, the students are expected to be totally safe.

\section{Conclusions}

A simple, robust, cheap and easy lab experiment to demonstrate quantum phenomena was developed in this work. In this sense, 145 students from biotechnology, pharmacy, engineers and geology courses have carried out the procedure in an undergraduate chemistry laboratory with no danger. Since the citric acid and CQDs are non-toxic materials. Therefore, the demonstration of the quantum phenomena including the light emission, scattering and absorption was successfully achieved. Once the procedure was completed by the students, the discussion begun and it was possible to note the excitation of the students. The visual effect provided by the experiments of light scattering and emission was able to kept the attention of the students, which it is very important to demonstrate the quantum mentioned phenomena. At the end of the lab experiment, the feedback of the students was positive. Moreover, materials including citric acid and the 3 in 1 pointer were used to compound the lab experiment. This keeps the lab experiment as cheap as possible. Therefore, chemistry laboratories observing lack of equipment can also demonstrate the quantum phenomena for their undergraduate students. Overall, CQDs can play as a powerful tool to demonstrate quantum phenomena.

\section{Supplemental Material}

A document including prelab, objective, introduction, experimental proedure and results sections is available for students and instructors from the corresponding author upon request.

\section{ACKNOWLEDGEMENTS}

This work was partly sponsored by CAPES, CNPq (Grants 478743/2013-0, O. P. F.) and FUNCAP (Brazilian agencies). Thanks to the Rubens Rodrigues for the assistance with the pictures displayed in the Fig. 3.

\section{REFERENCES}

[1] $\dagger$ Xiaoyou $\mathrm{Xu}$ et al., "Electrophoretic Analysis and Purification of Fluorescent Single-Walled Carbon Nanotube Fragments," J. Am. Chem. Soc., vol 126, no. 40, pp. 121736-12737, 2004.

[2] C. Fowley, B. McCaughan, A. Devlin, I. Yildiz, F. M. Raymo, and J. F. Callan, "Highly luminescent biocompatible carbon quantum dots by encapsulation with an amphiphilic polymer," Chem. Commun., vol. 48, no. 75, p. 9361, 2012.

[3] H. Ding, P. Zhang, T.-Y. Wang, J.-L. Kong, and H.-M. Xiong, "Nitrogen-doped carbon dots derived from polyvinyl pyrrolidone and their multicolor cell imaging," Nanotechnology, vol. 25, no. 20, p. 205604, 2014.

[4] S. Y. Lim et al., "Carbon quantum dots and their applications," Chem. Soc. Rev., vol. 44, no. 1, pp. 362-381, 2015.

[5] S.-T. Yang et al., "Carbon Dots for Optical Imaging in Vivo," J. Am. Chem. Soc., vol. 131, no. 32, pp. 11308-11309, 2009.

[6] Ya-Ping Sun et al., "Quantum-Sized Carbon Dots for Bright and Colorful Photoluminescence," J. Am. Chem. Soc., vol 128, no. 24 , pp. $7756-7757,2006$.

[7] R. Vaz et al., "Preparation of carbon dots and their optical characterization: an experiment of nanoscience for undergraduate course," Quim. Nova, vol. 38, no. 10, pp. 1366-1373, 2015.

[8] L. Pan et al., "Truly Fluorescent Excitation-Dependent Carbon Dots and Their Applications in Multicolor Cellular Imaging and Multidimensional Sensing," Adv. Mater., vol. 27, no. 47, pp. 7782-7787, 2015. 
[9] D. Pan et al., "Observation of $\mathrm{pH}-$, solvent-, spin-, and excitation-dependent blue photoluminescence from carbon nanoparticles," Chem. Commun., vol. 46, no. 21, p. 3681, 2010.

[10] S. Sahu, B. Behera, T. K. Maiti, and S. Mohapatra, "Simple one-step synthesis of highly luminescent carbon dots from orange juice: application as excellent bio-imaging agents," Chem. Commun., vol. 48, no. 70, p. 8835, 2012.

[11] S. Hu, A. Trinchi, P. Atkin, and I. Cole, "Tunable Photoluminescence Across the Entire Visible Spectrum from Carbon Dots Excited by White Light," Angew. Chemie Int. Ed., vol. 54, no. 10, pp. 2970-2974, 2015.

[12] Z.-H. Wen et al., "Excitation-independent carbon dots, from photoluminescence mechanism to single-color application," RSC Adv., vol. 6, no. 33, pp. 27829-27835, 2016.

[13] J. Hou et al., "Facile synthesis of carbon dots in an immiscible system with excitation-independent emission and thermally activated delayed fluorescence," Chem. Commun., vol. 51, no. 100, pp. 17768-17771, 2015.

[14] Y. Dong et al., "Carbon-Based Dots Co-doped with Nitrogen and Sulfur for High Quantum Yield and Excitation-Independent Emission," Angew. Chemie Int. Ed., vol. 52, no. 30, pp. 7800-7804, 2013.

[15] X. Li, S. Zhang, S. A. Kulinich, Y. Liu, and H. Zeng, "Engineering surface states of carbon dots to achieve controllable luminescence for solid-luminescent composites and sensitive $\mathrm{Be}^{2+}$ detection," Sci. Rep., vol. 4, pp. 161-164, 2014.

[16] H. Li et al., "Carbon nanodots: synthesis, properties and applications," J. Mater. Chem., vol. 22, no. 46, p. 24230, 2012.

[17] Z.-C. Yang et al., "Intrinsically fluorescent carbon dots with tunable emission derived from hydrothermal treatment of glucose in the presence of monopotassium phosphate," Chem. Commun., vol. 47, no. 42, p. 11615, 2011.

[18] M. L. Landry, T. E. Morrell, T. K. Karagounis, C.-H. Hsia, and C.-Y. Wang, "Simple Syntheses of CdSe Quantum Dots," J. Chem. Educ., vol. 91, no. 2, pp. 274-279, 2014.

[19] L. Cui, J. Wu, and H. Ju, "Electrochemical sensing of heavy metal ions with inorganic, organic and bio-materials," Biosens. Bioelectron., vol. 63, pp. 276-286, 2015.

[20] M. TUZEN, E. MELEK, and M. SOYLAK, "Celtek clay as sorbent for separation-preconcentration of metal ions from environmental samples," J. Hazard. Mater., vol. 136, no. 3, pp. 597-603, Aug. 2006.

[21] J. Mehta et al., "Progress in the biosensing techniques for trace-level heavy metals," Biotechnol. Adv., vol. 34, no. 1, pp. 47-60, 2016.

[22] H.-S. Lim, J.-S. Lee, H.-T. Chon, and M. Sager, "Heavy metal contamination and health risk assessment in the vicinity of the abandoned Songcheon $\mathrm{Au}-\mathrm{Ag}$ mine in Korea," $J$. Geochemical Explor., vol. 96, no. 2, pp. 223-230, 2008.

[23] F. Fu and Q. Wang, "Removal of heavy metal ions from wastewaters: A review," J. Environ. Manage., vol. 92, no. 3, pp. 407-418, 2011.

[24] G. F. Nordberg et al., "Prevalence of kidney dysfunction in humans - Relationship to cadmium dose, metallothionein, immunological and metabolic factors," Biochimie, vol. 91, no. 10, pp. 1282-1285, 2009.

[25] M. B. Arain et al., "Co-exposure of arsenic and cadmium through drinking water and tobacco smoking: Risk assessment on kidney dysfunction," Environ. Sci. Pollut. Res., vol. 22, no. 1, pp. 350-357, 2015.

[26] I. A. Mir, K. Das, K. Rawat, and H. B. Bohidar, "Hot injection versus room temperature synthesis of CdSe quantum dots: A differential spectroscopic and bioanalyte sensing efficacy evaluation," Colloids Surfaces A Physicochem. Eng. Asp., vol. 494, pp. 162-169, 2016.

[27] Y. Hou, Q. Lu, J. Deng, H. Li, and Y. Zhang, "One-pot electrochemical synthesis of functionalized fluorescent carbon dots and their selective sensing for mercury ion," Anal. Chim. Acta, vol. 866, pp. 69-74, 2015.

[28] Y. Dong, R. Wang, G. Li, C. Chen, Y. Chi, and G. Chen, "Polyamine-Functionalized Carbon Quantum Dots as Fluorescent Probes for Selective and Sensitive Detection of Copper Ions," Anal. Chem., vol. 84, no. 14, pp. 6220-6224, 2012.

[29] D. Pan, J. Zhang, Z. Li, and M. Wu, "Hydrothermal Route for Cutting Graphene Sheets into Blue - Luminescent Graphene Quantum Dots," Adv. Mater., vol. 22, no. 6, pp. 734-738, 2010 .

[30] L.-L. Li et al., "A Facile Microwave Avenue to Electrochemiluminescent Two-Color Graphene Quantum Dots," Adv. Funct. Mater., vol. 22, no. 14, pp. 2971-2979, 2012.

[31] I. Costas-Mora, V. Romero, I. Lavilla, and C. Bendicho, "In Situ Building of a Nanoprobe Based on Fluorescent Carbon Dots for Methylmercury Detection," Anal. Chem., vol. 86, no. 9, pp. 4536-4543, 2014.

[32] Y. Dong et al., "Blue luminescent graphene quantum dots and graphene oxide prepared by tuning the carbonization degree of citric acid," Carbon N. Y., vol. 50, no. 12, pp. 4738-4743, 2012.

[33] J. Schneider et al., "Molecular Fluorescence in Citric Acid-Based Carbon Dots," J. Phys. Chem. C, vol. 121, no. 3, pp. 2014-2022, 2017.

[34] L. Wang and H. S. Zhou, "Green Synthesis of Luminescent Nitrogen-Doped Carbon Dots from Milk and Its Imaging Application," Anal. Chem., vol. 86, no. 18, pp. 8902-8905, 2014.

[35] Q. Liang, W. Ma, Y. Shi, Z. Li, and X. Yang, "Easy synthesis of highly fluorescent carbon quantum dots from gelatin and their luminescent properties and applications," Carbon N. Y., vol. 60, pp. 421-428, 2013.

[36] C. Jiang, H. Wu, X. Song, X. Ma, J. Wang, and M. Tan, "Presence of photoluminescent carbon dots in Nescafe $\AA$ original instant coffee: Applications to bioimaging," Talanta, vol. 127, pp. 68-74, 2014.

[37] D. Sun, R. Ban, P.-H. Zhang, G.-H. Wu, J.-R. Zhang, and J.-J. $\mathrm{Zhu}$, "Hair fiber as a precursor for synthesizing of sulfur- and nitrogen-co-doped carbon dots with tunable luminescence properties," Carbon N. Y., vol. 64, pp. 424-434, 2013.

[38] B. De et al., "A green and facile approach for the synthesis of 
water soluble fluorescent carbon dots from banana juice," RSC Adv., vol. 3, no. 22, p. 8286, 2013.

[39] B. Chen et al., "Large scale synthesis of photoluminescent carbon nanodots and their application for bioimaging," Nanoscale, vol. 5, no. 5, p. 1967, 2013.

[40] C. Liu et al., "One-step synthesis of surface passivated carbon nanodots by microwave assisted pyrolysis for enhanced multicolor photoluminescence and bioimaging," J. Mater. Chem., vol. 21, no. 35, p. 13163, 2011.

[41] S. Wang, Z.-G. Chen, I. Cole, and Q. Li, "Structural evolution of graphene quantum dots during thermal decomposition of citric acid and the corresponding photoluminescence," Carbon N. Y., vol. 82, pp. 304-313, 2015.

[42] T. Oppenländer, "Glossary of Terms Used in Photochemistry," in Photochemical Purification of Water and Air, Weinheim, Germany: Wiley-VCH Verlag GmbH \& Co. KGaA, pp. 299-352, 2007.

[43] S. E. Braslavsky et al., "Glossary of terms used in photochemistry 3 rd edition (IUPAC Recommendations 2006)," Pure Appl. Chem, vol. 79, no. 3, pp. 293-465, 2007.

[44] J. Shen, S. Gagliardi, M. R. S. McCoustra, and V. Arrighi, "Effect of humic substances aggregation on the determination of fluoride in water using an ion selective electrode," Chemosphere, vol. 159, pp. 66-71, 2016.

[45] H. Ding, S.-B. Yu, J.-S. Wei, and H.-M. Xiong, "Full-Color Light-Emitting Carbon Dots with a Surface-State-Controlled Luminescence Mechanism," ACS Nano, vol. 10, no. 1, pp. 484-491, 2016.
[46] L. Wang et al., "Common Origin of Green Luminescence in Carbon Nanodots and Graphene Quantum Dots," ACS Nano, vol. 8, no. 3, pp. 2541-2547, 2014.

[47] S. Zhu et al., "Surface Chemistry Routes to Modulate the Photoluminescence of Graphene Quantum Dots: From Fluorescence Mechanism to Up-Conversion Bioimaging Applications," Adv. Funct. Mater., vol. 22, no. 22, pp. 4732-4740, 2012.

[48] S. Zhu, Y. Song, X. Zhao, J. Shao, J. Zhang, and B. Yang, "The photoluminescence mechanism in carbon dots (graphene quantum dots, carbon nanodots, and polymer dots): current state and future perspective," Nano Res., vol. 8, no. 2, pp. 355-381, 2015.

[49] Dan Qu et al., "Highly luminescent S, N co-doped graphene quantum dots with broad visible absorption bands for visible light photocatalysts," Nanoscale, vol. 5, no. 24, pp. 12272-12277, 2013.

[50] F. T. Thema et al., "Synthesis and Characterization of Graphene Thin Films by Chemical Reduction of Exfoliated and Intercalated Graphite Oxide," J. Chem., vol. 2013, pp. $1-6,2013$.

[51] Z. Gan et al., "Mechanism for excitation-dependent photoluminescence from graphene quantum dots and other graphene oxide derivates: consensus, debates and challenges," Nanoscale, vol. 8, no. 15, pp. 7794-7807, 2016.

[52] H. Dang, L.-K. Huang, Y. Zhang, C.-F. Wang, and S. Chen, "Large-Scale Ultrasonic Fabrication of White Fluorescent Carbon Dots," Ind. Eng. Chem. Res., vol. 55, no. 18, pp. 5335-5341, 2016. 